\title{
Fluorescence resonant energy transfer in the optical near field
}

\author{
Gérard Colas des Francs and Christian Girard* \\ Centre d'Elaboration des Matériaux et d'Etudes Structurales (CNRS), 29 rue Jeanne Marvig, F-31055 Toulouse, France \\ Olivier J. F. Martin \\ Electromagnetic Fields and Microwave Electronics Laboratory ETHZ, Gloriastrasse 35, 8092 Zurich, Switzerland
}

(Received 6 September 2002; published 27 May 2003)

\begin{abstract}
We develop a versatile theoretical framework for the study of fluorescence resonant energy transfer (FRET, or Förster transfer) in complex environments, under arbitrary illumination, including optical near fields. By combining the field-susceptibility formalism with the optical Bloch equations method, we derive general equations for the computation of the energy transfer between pairs of donor-acceptor molecules excited by optical near fields and placed in a complex geometry. This approach allows accounting for both the variations of the molecular population rates and the influence of the environment. Several examples illustrate the ability of the technique to analyze recent FRET experiments performed in the optical near field.
\end{abstract}

DOI: 10.1103/PhysRevA.67.053805

PACS number(s): 42.50.Ct, 07.79.Fc, 33.80.-b, 87.64.Xx

\section{INTRODUCTION}

The fluorescence resonant energy transfer (FRET) involves a nonradiative dipole-dipole coupling between an excited fluorescent donor molecule and a fluorescent acceptor molecule (Förster transfer) [1]. This photoinduced energy transfer mechanism has been extensively studied in the past, particularly for its contribution to some photosynthesis mechanisms [2,3], as well as in light-emitting diode devices $[4,5]$. It has also been observed in living cells, and provided new insights into specific cellular phenomena such as protein interaction [6-8].

In the context of near-field optics (NFO), the strong dependence of the energy transfer rate on the donor-acceptor distance opens new and interesting perspectives, as the fluorescence can be locally excited or detected with a scanning near-field optical microscope (SNOM) [9-14]. SNOM belongs to the family of local probe microscopes that use optical evanescent waves to overpass the usual diffraction limit associated with conventional far-field microscopes. Figure 1 shows a schematic experimental configuration that can be used to trigger FRET in the optical near field. A SNOM tip is raster scanned over a sample containing both fluorescent donor and fluorescent acceptor molecules deposited on a surface. Only the donors that are in close proximity of the illuminating tip are excited. The FRET then occurs between the donor molecules in the excited state and the acceptor molecules in the ground state. This configuration makes therefore possible a local investigation of FRET.

SNOM further allows the simultaneous measurement of the topographical structure and the optical properties of the system. In a realistic experiment, the near-field optical excitation of dye molecules occurs within complex surroundings, so that the fluorescence properties of the chromophores are significantly altered. The interaction between donor and acceptor chromophores is also modified by the presence of this complex environment. For example, the presence of the sur-

*Email address: girard@cemes.fr face supporting the molecules, or the bulky SNOM tip, or even the cavity formed between the tip and the surface, strongly modifies the fluorescence lifetime of each chromophore [15-18] as well as the dipole-dipole coupling between them [19]. In this way, the FRET energy transfer rate may be enhanced or reduced, depending on the coupling strength between the molecules and their surroundings $[20,21]$.

To analyze such configurations involving highly confined optical near fields and molecular resonant processes, we propose in the present work to combine two well developed numerical techniques; namely, the field-susceptibility or Green-function formalism [22] and the optical Bloch equations method [23]. With the former, parameters such as dipole-dipole coupling and fluorescence lifetimes in a confined geometry can be deduced from the field susceptibility associated with that geometry. These parameters can then be introduced in the optical Bloch equations to describe the molecular population levels and to obtain both the donor and acceptor fluorescence signals.

Let us note that a related approach based on the Schrödinger equation coupled to Maxwell's equations, was recently presented for the excitonic regime [24]. In this case, the tip and sample were treated quantum mechanically as an ensemble of organic molecules with a single-resonant energy level and a transient dipole moment. In the numerical applications to be discussed in the present paper, we adopt a description where the active part of the tip (the very tip) is modeled as an illuminating dipolar source, while the physical tip (the tip body) is treated as a dielectric pyramid coated with a thin metallic layer. This choice was motivated by the quality of previous theoretical analyses of SNOM images using a pointlike emitter.

The paper is organized as follows: The theoretical framework is developed in Sec. II, where the optical Bloch equations are introduced and the formulas for the fluorescence signal are derived. In Sec. III, examples of increasing complexity illustrate the utilization of the technique for the simulation of experimental configurations. A summary and outlook is given in Sec. IV. 


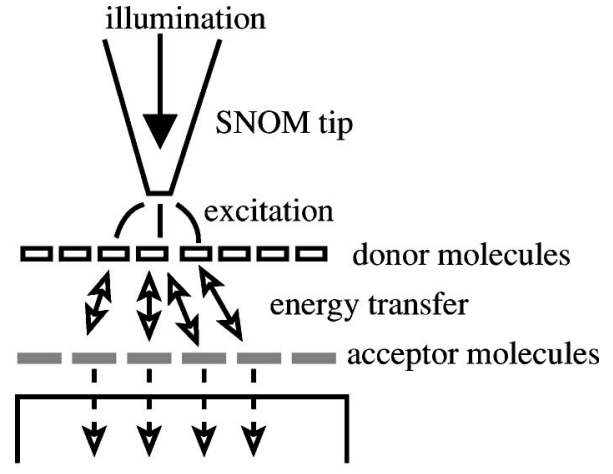

fluorescence detection

FIG. 1. Experimental setup, the donor molecules are locally excited with a SNOM tip. Then, energy transfer occurs towards acceptor molecules in proximity of the excited donor molecules. The fluorescent signals of both donor and acceptor fluorescent dyes are detected below the surface.

\section{FORMALISM}

\section{A. The optical Bloch equations}

Since it allows an easy selection of the active molecular levels, the matrix density formalism provides a flexible framework to describe the internal dynamics of two chromophores interacting with an external optical field [23]. The optical Bloch equations built from the population evolution of these active quantum states provide all the ingredients required to describe FRET in a confined geometry. Furthermore, the resulting occupation state representation avoids conceptual difficulties that usually occur with a treatment based on a self-consistent linear response theory between the two active molecules. In particular, the matrix density formalism allows fluorescence saturation effects to be introduced without any formal difficulties.

In this section, we develop this formalism using a approach similar to that used previously to investigate fluorescence enhancement in the optical near field [25], or the van der Waals dispersive interactions in a two-dimensional cavity [26].

\section{Interaction Hamiltonian}

Let us consider two dye molecules, called donor and acceptor located, respectively, at the position vectors $\mathbf{r}_{1}$ and $\mathbf{r}_{2}$ (Fig. 2). In the presence of a Förster-like transfer, the incident optical electric field (wavelength $\lambda_{l}=2 \pi c / \omega_{l}$ ) is used to excite an electronic transition near the resonant wavelength $\left(\lambda_{1}=2 \pi c / \omega_{1}=380 \mathrm{~nm}\right)$ of the donor molecule. After relaxation to the first vibrational excited level $\left|a_{1}\right\rangle$, this molecule is then coupled via a dipole-dipole process to the excited electronic level $\left|b_{2}\right\rangle$ with the same energy of the acceptor molecule. Finally after relaxation, the acceptor molecule goes back into its ground state by emitting a longer wavelength photon $\left(\lambda_{2}=2 \pi c / \omega_{2}=500 \mathrm{~nm}\right)$. Let us note that level $\left|a_{1}\right\rangle$ can also directly relax by emitting a photon of wavelength $\lambda_{0}=2 \pi c / \omega_{0}=425 \mathrm{~nm}$, with a fluorescence rate $\Gamma_{a 1}$.

For sake of clarity, we only indicate the excited states of the molecular pair. For instance, the state $\left|a_{1} g_{2}\right\rangle$ is noted by

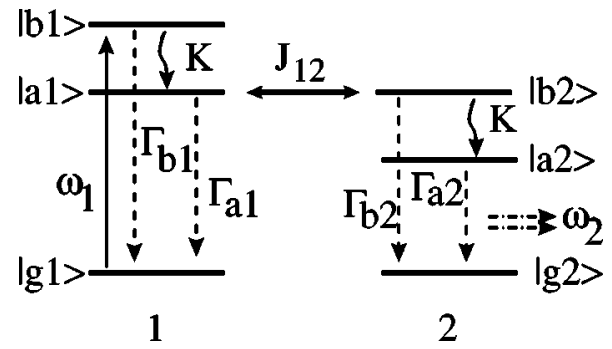

FIG. 2. Schematic representation of active molecular levels of the pair of donor (dye 1) and acceptor (dye 2) molecules. The two quantum states $|a 1\rangle$ and $|b 2\rangle$ are coupled by a resonant dipolar interaction mechanism characterized by the $J_{12}$ interaction parameter.

$\left|a_{1}\right\rangle$. The ground state of the pair of molecule is noted by $|g\rangle=\left|g_{1} g_{2}\right\rangle$. With these notations, the chromophores pair can be described with the following Hamiltonian $H$ :

$$
H=H_{0}+W(t)+H_{d d},
$$

with

$H_{0}=E_{a_{1}}\left|a_{1}\right\rangle\left\langle a_{1}\left|+E_{b_{1}}\right| b_{1}\right\rangle\left\langle b_{1}\left|+E_{b_{2}}\right| b_{2}\right\rangle\left\langle b_{2}\left|+E_{a_{2}}\right| a_{2}\right\rangle\left\langle a_{2}\right|$

and

$$
\begin{aligned}
H_{d d}= & J_{12}\left|a_{1}\right\rangle\left\langle b_{2}\left|+J_{12}^{\star}\right| b_{2}\right\rangle\left\langle a_{1}\right| \\
= & \hbar \Omega_{12}\left\{\left|a_{1}\right\rangle\left\langle a_{2}|+| b_{2}\right\rangle\left\langle a_{1}\right|\right\} \\
& -i \hbar \gamma_{12}\left\{\left|a_{1}\right\rangle\left\langle b_{2}|-| b_{2}\right\rangle\left\langle a_{1}\right|\right\},
\end{aligned}
$$

where $E_{\alpha}$ represents the energy level $\alpha, W(t)$ is the coupling Hamiltonian between the laser excitation and molecule 1, and $H_{d d}$ is the interaction Hamiltonian between the two dyes. In the framework of the dipole-dipole coupling approximation, this Hamiltonian can be rewritten by introducing the coupling strength $\Omega_{12}$ and the cooperative decay $\gamma_{12}$ [Eq. (4)]. We only consider one single-resonant term, but this approach can easily be generalized to include the spectral profiles of both the dyes in a phenomenological way.

In Eqs. (1)-(4), the FRET coupling strength is hidden in the $J_{12}$ factor. As emphasized in several previous papers, the environment directly influences this coupling factor $[19,27,28]$. Electrodynamics theory demonstrates that this environment's response is fully included in the fieldsusceptibility tensor $\mathbf{S}\left(\mathbf{r}, \mathbf{r}^{\prime}, \omega\right)$ [29], defined from the electric field produced at $\mathbf{r}$ by a fluctuating dipole $\mathbf{p}$ located at $\mathbf{r}^{\prime}$, oscillating with the frequency $\omega$ :

$$
\mathbf{E}(\mathbf{r}, t)=\operatorname{Re}\left\{\mathbf{S}\left(\mathbf{r}, \mathbf{r}^{\prime}, \omega\right) \cdot \mathbf{p}(\omega) e^{-i \omega t}\right\}
$$

In a general way, $\mathbf{S}\left(\mathbf{r}, \mathbf{r}^{\prime}, \omega\right)$ is deduced from Maxwell's equations [30]. The dipole-dipole coupling term then reads [31]

$$
J_{12}=-\mathbf{p}_{2} \cdot \mathbf{S}\left(\mathbf{r}_{2}, \mathbf{r}_{1}, \omega_{0}\right) \cdot \mathbf{p}_{1},
$$


where $\mathbf{p}_{1}$ and $\mathbf{p}_{2}$, are the dipole transition moments associated with the electronic transitions between $\left|a_{1}\right\rangle$ and $\left|g_{1}\right\rangle$, $\left|b_{2}\right\rangle$ and $\left|g_{2}\right\rangle$, respectively. In the following, the coupling factor $J_{12}$ will be rewritten as

$$
J_{12}=\hbar\left[\Omega_{12}-i \gamma_{12}\right],
$$

where we separated the dipole-dipole strength $\Omega_{12}$ and the cooperative decay rate $\gamma_{12}[19]$.

\section{Evolution equations}

For the illumination, we assume that the pair of dyes is excited with an arbitrary monochromatic optical electric near-field distribution $\mathbf{E}(\mathbf{r}, t)$. In spite of its evanescent character, this field can efficiently excite a fluorescent molecule. Although such an optical near field can be confined on subwavelength volumes [32], its spatial variation over the extension of a single molecule remains moderate, so that the dipolar approximation can be used to model the excitation.

For the Förster transfer we make the following hypotheses.

(i) The illumination of optical near field only couples the excited level $\left|b_{1}\right\rangle$ of the first dye, located at the position $\mathbf{r}_{1}$. The coupling Hamiltonian reduces to

$$
W(t)=-\hat{\boldsymbol{\mu}}_{1} \cdot \mathbf{E}\left(\mathbf{r}_{1}, t\right),
$$

with

$$
\hat{\boldsymbol{\mu}}_{1}=\boldsymbol{\mu}_{1}\left\{|g\rangle\left\langle b_{1}|+| b_{1}\right\rangle\langle g|\right\},
$$

where $\boldsymbol{\mu}_{1}$ denotes the transition dipole moment between the ground state $\left|g_{1}\right\rangle$ and the excited level $\left|b_{1}\right\rangle$ of the donor fluorescent molecule. We further assume that the optical electric field viewed by the donor molecule is harmonic. In a general way, both phase and amplitude of the optical near field strongly depend on the observation point $\mathbf{r}_{1}$. One has then

$$
\mathbf{E}\left(\mathbf{r}_{1}, t\right)=\operatorname{Re}\left\{\mathbf{E}_{0}\left(\mathbf{r}_{1}\right) e^{-i\left(\omega_{l} t+\Phi\left(\mathbf{r}_{1}\right)\right)}\right\} .
$$

(ii) In addition to the two coupling mechanisms already discussed, namely, the illumination-molecule 1 interaction [term $W(t)$ in Eq. (1)] and the dipole-dipole interaction [term $H_{d d}$ in Eq. (1)], two mechanisms must now be introduced in a phenomenological way. First, the coupling between environment and molecules is responsible for spontaneous decay. After averaging on the surrounding states, it can be represented with the Redfield operator $\mathcal{R}_{\text {spont }}$ acting on the molecules pair density matrix operator $\rho$. Second, the interaction with the vibrational bath leads to nonradiative vibrational decay. After averaging on the bath modes, it can also be represented with a second Redfield operator $\mathcal{R}_{v i b}$. These four coupling mechanisms have very different characteristic times (correlation times). Therefore, the independent speed variation approximation is suitable and the four mechanisms can be treated independently [23]. With these assumptions, the Schrödinger representation of the Liouville equation leads to

$$
\begin{aligned}
\frac{d}{d t} \rho(t)= & \frac{1}{i \hbar}\left[H_{0}, \rho(t)\right]+\frac{1}{i \hbar}\left[H_{d d}, \rho(t)\right] \\
& +\frac{1}{i \hbar}[W(t), \rho(t)]+\mathcal{R}_{\text {spont }} \rho(t)+\mathcal{R}_{v i b} \rho(t) .
\end{aligned}
$$

In the presence of the Förster transfer, the magnitude of the dipole-dipole coupling $\left|J_{12}\right|$ is assumed to be weak compared to $\hbar \Gamma_{a_{1}}$, so that it can be treated as a perturbation. In the case of stronger dipole-dipole coupling, this approximation is not anymore valid and an excitonic model must be used [24]. Additionally, Eq. (11) is not valid when $H_{d d}$ is nonHermitian. In this case, the cooperative decay should be considered, which renders the solution of the corresponding Liouville equation more complicated.

(iii) We apply the usual rotating waves approximation that neglects nonresonant terms in the evolution process and yields the optical Bloch equations derived from the Liouville equation (11):

$$
\begin{aligned}
\frac{d}{d t} \rho_{a_{2} a_{2}}(t)= & K \rho_{b_{2} b_{2}}(t)-\Gamma_{a_{2}} \rho_{a_{2} a_{2}}(t), \\
\frac{d}{d t} \rho_{b_{2} b_{2}}(t)= & -\left(K+\Gamma_{b_{2}}\right) \rho_{b_{2} b_{2}}(t) \\
& +i \Omega_{12}\left[\rho_{b_{2} a_{1}}(t)-\rho_{a_{1} b_{2}}(t)\right],
\end{aligned}
$$

$$
\begin{aligned}
\frac{d}{d t} \rho_{b_{2} a_{1}}(t)= & -\gamma_{b_{2} a_{1}} \rho_{b_{2} a_{1}}(t) \\
& +i \Omega_{12}\left[\rho_{b_{2} b_{2}}(t)-\rho_{a_{1} a_{1}}(t)\right], \\
\frac{d}{d t} \rho_{a_{1} b_{2}}(t)= & -\gamma_{b_{2} a_{1}} \rho_{a_{1} b_{2}}(t) \\
& -i \Omega_{12}\left[\rho_{b_{2} b_{2}}(t)-\rho_{a_{1} a_{1}}(t)\right], \\
\frac{d}{d t} \rho_{a_{1} a_{1}}(t)= & K \rho_{b_{1} b_{1}}(t)-\Gamma_{a_{1}} \rho_{a_{1} a_{1}}(t) \\
& -i \Omega_{12}\left[\rho_{b_{2} a_{1}}(t)-\rho_{a_{1} b_{2}}(t)\right],
\end{aligned}
$$

$$
\begin{aligned}
\frac{d}{d t} \rho_{b_{1} b_{1}}(t)= & -\left(K+\Gamma_{b_{1}}\right) \rho_{b_{1} b_{1}}(t) \\
& +i \frac{\Omega}{2}\left[\rho_{b_{1} g}(t)-\rho_{g b_{1}}(t)\right],
\end{aligned}
$$

$$
\begin{aligned}
\frac{d}{d t} \rho_{b_{1} g}(t)= & \left(i \delta \omega-\gamma_{b_{1} g}\right) \rho_{b_{1} g}(t) \\
& -i \frac{\Omega}{2}\left[\rho_{g g}(t)-\rho_{b_{1} b_{1}}(t)\right],
\end{aligned}
$$

$$
\begin{aligned}
\frac{d}{d t} \rho_{g b_{1}}(t)= & -\left(i \delta \omega+\gamma_{b_{1} g}\right) \rho_{g b_{1}}(t) \\
& +i \frac{\Omega}{2}\left[\rho_{g g}(t)-\rho_{b_{1} b_{1}}(t)\right],
\end{aligned}
$$

where we have introduced the Rabi pulsation $\Omega$, 


$$
\Omega=-\frac{\boldsymbol{\mu}_{1} \cdot \mathbf{E}_{0}\left(\mathbf{r}_{1}\right)}{\hbar}
$$

and the detuning $\delta \omega$ between the laser and the resonant absorption frequency of molecule 1 ,

$$
\delta \omega=\omega_{l}-\omega_{1}
$$

The quantity $K$ represents the vibrational relaxation constant of both $\left|b_{1}\right\rangle$ and $\left|b_{2}\right\rangle$ levels. This parameter is chosen identical for the two chromophores. The other parameters $\Gamma_{\alpha}$ and $\gamma_{\alpha \beta}$ represent the fluorescence width of the excited level $\alpha$ and the coherence decay rate associated with the $(\alpha, \beta)$ transition, respectively. These parameters appear in the optical Bloch equations after the application of the Redfield operator on the matrix density operator; in the present case, symmetry considerations imply that several Redfield operator matrix elements vanish [23].

The coherence decay rates $\gamma_{\alpha \beta}$ in Bloch equations (12)(19) can be related to the fluorescence width of the excited levels [23]:

$$
\begin{aligned}
\gamma_{b_{2} a_{1}} & =\left(\Gamma_{b_{2}}+\Gamma_{a_{1}}\right) / 2, \\
\gamma_{b_{1} g} & =\Gamma_{b_{1}} / 2 .
\end{aligned}
$$

\section{Fluorescence signals}

The stationary populations of the $\left|a_{1}\right\rangle$ and $\left|a_{2}\right\rangle$ levels give direct access to the detected fluorescence signal. Furthermore, the density operator satisfies the condition

$$
\rho_{g g}+\rho_{a_{1} a_{1}}+\rho_{b_{1} b_{1}}+\rho_{a_{2} a_{2}}+\rho_{b_{2} b_{2}}=1 \text {. }
$$

This stationary regime of energy exchange between the different paths is reached when all the terms on the left-hand side of Bloch equations (12)-(19) tend simultaneously to zero. Together with Eq. (24), this procedure leads to

$$
\begin{aligned}
\rho_{a_{1} a_{1}}= & \rho_{a_{1} a_{1}}^{(0)}-2\left[\rho_{a_{1} a_{1}}^{(0)}\right]^{2}\left[1+\frac{K}{\Gamma_{a_{2}}}+\frac{2\left(K+\Gamma_{b_{2}}\right)}{K}\left(1+\frac{1}{s}\right)\right] \\
& \times \frac{\Omega_{12}^{2}}{\left(\Gamma_{b_{2}}+K\right) \gamma_{b_{2} a_{1}}}
\end{aligned}
$$

and

$$
\rho_{a_{2} a_{2}}=\rho_{a_{1} a_{1}}^{(0)} \frac{2 K\left|\Omega_{12}\right|^{2}}{\left(K+\Gamma_{b_{2}}\right) \Gamma_{a_{2}} \gamma_{a_{1} b_{2}}}
$$

where we have introduced the donor population $\rho_{a_{1} a_{1}}^{(0)}$ of the $\left|a_{1}\right\rangle$ level in the absence of the Förster transfer,

$$
\rho_{a_{1} a_{1}}^{(0)}=\frac{(\alpha-1) s}{1+\alpha s}
$$

and defined the parameters $\Omega_{12}$,

$$
\Omega_{12}=\operatorname{Re}\left[\frac{J_{12}}{\hbar}\right]
$$

and $\alpha$,

$$
\alpha=1+K /\left(2 \Gamma_{a_{1}}\right)
$$

as well as the saturation parameter $s$,

$$
s=\frac{\gamma_{b_{1} g 1}}{K+\Gamma_{b_{1}}} \frac{\Omega^{2}}{\delta \omega^{2}+\gamma_{b_{1} g 1}^{2}} .
$$

All the ingredients required to describe the total fluorescence signals generated both by the donor and the acceptor dyes are now available [23]:

$$
\begin{gathered}
I_{\text {donor }}\left(\omega_{0}\right)=\hbar \omega_{0} \Gamma_{a_{1}} \rho_{a_{1} a_{1}}, \\
I_{\text {acceptor }}\left(\omega_{2}\right)=\hbar \omega_{2} \Gamma_{a_{2}} \rho_{a_{2} a_{2}} .
\end{gathered}
$$

Equations (31) and (32) allow to explicitly compute the fluorescence signal measured for each molecule, at its corresponding emission frequencies $\omega_{0}$ and $\omega_{2}$, in a Förster transfer experiment.

\section{B. Generalization to several donor-acceptor pairs}

Most of the FRET experiment measurements involve a large number of fluorescent donor and acceptor pairs, organized, for example, in different layers [9]. To describe such a situation, we must generalize the previous formalism to multiple donor-acceptor interactions. We first consider a singledonor dye, coupled to $N$ acceptor molecules. Since weak coupling is assumed, each acceptor perturbs the donor fluorescence signal only weakly and Eqs. (25) and (26) can be generalized to give

$$
\begin{aligned}
\rho_{a_{1} a_{1}}= & \rho_{a_{1} a_{1}}^{(0)}-2\left[\rho_{a_{1} a_{1}}^{(0)}\right]^{2} \sum_{j=1}^{N}\left[1+\frac{K}{\Gamma_{a_{2}}}+\frac{2\left(K+\Gamma_{b_{2}}\right)}{K}\right. \\
& \left.\times\left(1+\frac{1}{s}\right)\right] \frac{\Omega_{1, j}^{2}}{\left(\Gamma_{b_{2}}+K\right) \gamma_{b_{2} a_{1}}},
\end{aligned}
$$

and

$$
\rho_{a_{2} a_{2}}(j)=\rho_{a_{1} a_{1}}^{(0)} \frac{2 K \Omega_{1, j}^{2}}{\left(K+\Gamma_{b_{2}}\right) \Gamma_{a_{2}} \gamma_{b_{2} a_{1}}}, \quad j=1, \ldots, N,
$$

where $\Omega_{1, j}$ represents the coupling constant between the donor fluorescent molecule and the $j$ th acceptor dye.

In the presence of $M$ donor molecules, the fluorescence intensities can be incoherently added up: 


$$
\begin{aligned}
I\left(\omega_{0}\right) \propto & \Gamma_{a_{1}} \sum_{i=1}^{M} \sigma_{i}-2 \sigma_{i}^{2}\left[1+\frac{K}{\Gamma_{a_{2}}}+\frac{2\left(K+\Gamma_{b_{2}}\right)}{K}\left(1+\frac{1}{s(i)}\right)\right] \\
& \times \frac{J(i)}{\left(\Gamma_{b_{2}}+K\right) \gamma_{b_{2} a_{1}}},
\end{aligned}
$$

and

$$
I\left(\omega_{2}\right) \propto \sum_{i}^{M} \sigma_{i} \frac{2 K J(i)}{\left(K+\Gamma_{b_{2}}\right) \gamma_{b_{2} a_{1}}},
$$

with

$$
\sigma_{i}=\frac{(\alpha-1) s(i)}{1+\alpha s(i)}
$$

and

$$
J(i)=\sum_{j=1}^{M} \Omega_{i, j}^{2}, \quad i=1, \ldots, M .
$$

In these last equations, we have explicitly indicated the dependence on the $i$ th donor molecule. Similarly like what was done in the previous section, the factor $\Omega_{i, j}^{2}$ denotes the coupling constant between the $i$ th donor molecule and the $j$ th acceptor molecule.

\section{RESULTS}

\section{A. Optical saturation}

To check the predictability of our model, we first present some simple simulations involving only one donor-acceptor pair, deposited on a surface and illuminated by a dipolar source located at the position $\mathbf{r}_{t i p}=\left(x_{t i p}, y_{t i p}, z_{t i p}\right)$. (The case of many donor-acceptor pairs is considered in Sec. III B and the influence of a massive illumination tips in Sec. III C.)

In this first application, as well as the others discussed in this paper, the illumination field generated by the SNOM probe is assumed to be dipolar. We assume the orientation of this illumination dipole parallel to the $O x$ axis. The two dye molecules are chosen with the same orientation (inset in Fig. 3). The magnitude of their dipole transition moment has been calculated with an oscillator strength

$$
f_{i}=2 m \omega_{i}\left|\mu_{i}\right|^{2} / e^{2} \hbar=0.1,
$$

where $e$ and $m$ are the electron charge and mass. The following data have been used to describe the excited level widths and the vibrational relaxation constants of both isolated chromophores: $\Gamma_{i}=10^{12} \mathrm{~s}^{-1}, K=10^{15} \mathrm{~s}^{-1}$.

Figure 3 shows the excitation spectra computed from Eq. (31). Two different emitted powers are considered $(0.1 \mathrm{nW}$ and $0.1 \mu \mathrm{W})$. Because of the nonperturbative treatment inherent to the use of the optical Bloch equations, the phenomenon of optical saturation is well reproduced. It manifests itself in a significant broadening of the excitation spectrum when the excitation power increases.

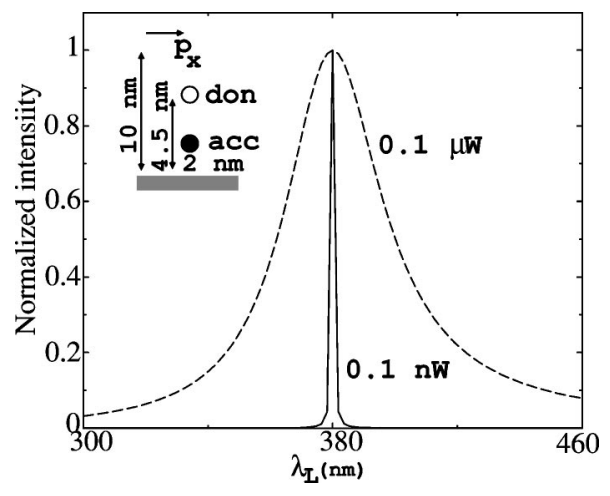

FIG. 3. Excitation spectra computed from Eq. (31) for two different excitation powers. The positions of the fluorescent dyes and the orientation of the exciting dipole are given in the inset.

\section{B. FRET images simulations}

In this section, we present computer simulations of FRET images based on the numerical implementation of Eqs. (35) and (36). The configuration is inspired from several recent FRET experiments [9-14]. The donor and acceptor molecules are deposited in different layers on a glass surface and a small SNOM tip is used to excite the donor fluorescent molecules in the near field [Fig. 4(a)].

The following hypotheses are made for the calculation:

(i) The illuminating probe tip is represented as a pointlike dipolar source oriented along the (OX) axis [Fig. 4(a)]. This choice is motivated by previous studies that clearly indicated that most SNOM tips behave like a dipolar light source parallel to the surface plane [15,33-35]. (The influence of a more massive tip on the FRET images will, however, be investigated in Sec. III C.)

(ii) The electrostatic limit is taken for the dipole-dipole coupling factor, which includes both the free-space propagator [through the dyadic tensor $\mathbf{T}\left(\mathbf{r}_{1}, \mathbf{r}_{2}\right)$ ] and the coupling to the complex surrounding [through the dyadic tensor $\left.\mathbf{S}_{\mathbf{s}}\left(\mathbf{r}_{1}, \mathbf{r}_{2}, \omega_{0}\right)\right]$. The validity of this approximation for NFO has been discussed in Ref. [36]. This leads to

$$
J_{12}=-\mathbf{p}_{2} \cdot\left[\mathbf{T}\left(\mathbf{r}_{2}, \mathbf{r}_{1}\right)+\mathbf{S}_{\mathbf{s}}\left(\mathbf{r}_{2}, \mathbf{r}_{1}, \omega_{0}\right)\right] \cdot \mathbf{p}_{1}
$$

with

$$
\mathbf{T}\left(\mathbf{r}_{1}, \mathbf{r}_{2}\right)=\frac{1}{4 \pi \epsilon_{0}} \frac{3\left(\mathbf{r}_{1}-\mathbf{r}_{2}\right)\left(\mathbf{r}_{1}-\mathbf{r}_{2}\right)-\left|\mathbf{r}_{1}-\mathbf{r}_{2}\right|^{2} \mathbf{I}}{\left|\mathbf{r}_{1}-\mathbf{r}_{2}\right|^{5}}
$$

and

$$
\mathbf{S}_{s}\left(\mathbf{r}_{1}, \mathbf{r}_{2}, \omega\right)=\mathbf{T}\left(\mathbf{r}_{1}, \mathbf{r}_{2}\right) \frac{\epsilon_{s}(\omega)-\epsilon_{0}}{\epsilon_{s}(\omega)+\epsilon_{0}}\left[\begin{array}{ccc}
-1 & 0 & 0 \\
0 & -1 & 0 \\
0 & 0 & 1
\end{array}\right],
$$

where $\epsilon_{s}(\omega)$ represents the dielectric permittivity of the surface.

(iii) The different level decay rates $\Gamma_{i}^{0}$ are introduced in Eqs. (35) and (36) by neglecting the surrounding changes, using the standard relation 

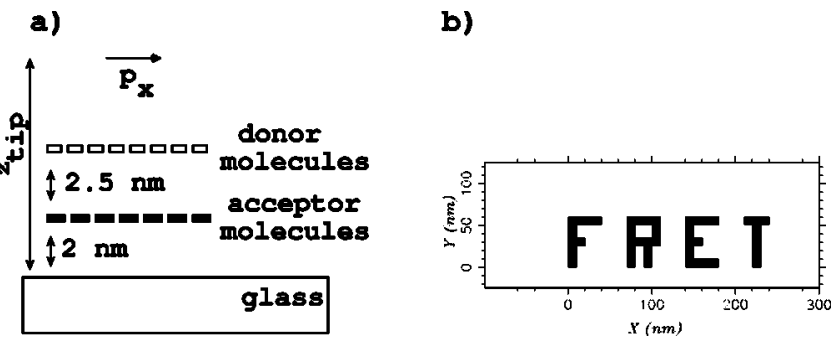

FIG. 4. (a) Experimental setup, a dipolar-light source excites the donor molecules which then couple to the acceptor molecules. The fluorescence signals are detected under the surface. Both donor and acceptor molecules are placed at the nodes of a square lattice, with a regular spacing of $1 \mathrm{~nm}$, and $2 \mathrm{~nm}$, respectively. (b) Top view illustrating the pattern created by the acceptor molecules.

$$
\Gamma_{i}^{0}=\frac{\omega_{i}^{3}}{3 \pi \epsilon_{0} \hbar c^{3}}\left|\mu_{i}\right|^{2}
$$

where $\mu_{i}$ is the electronic dipole moment magnitude of the level $i$.

The illumination dipole amplitude has been adjusted to reach a radiated power of $0.1 \mathrm{nW}$ at the wavelength $\lambda_{l}$ $=380 \mathrm{~nm}$. The fluorescent molecular parameters and orientations are identical to those previously used in Sec. III A. Furthermore, we assume that the acceptor molecules have been deposited on the glass surface by patterning the acronym FRET with nanometric lateral dimensions [Fig. 4(b)]. From a practical point of view, such patterns can be written by selectively photodestructing the acceptor dyes in predefined regions. This can be made in the near field with the light delivered by the tip of the microscope itself $[10,37]$ or by using a nanoimprint technique [38]. The donor molecules are then spread out over the acceptor layer, using a weaker molecular concentration.

Figure 5 shows two images of the fluorescent signals, obtained by summing up the incoherent intensities at a given wavelength [Eqs. (35) and (36)]. Figure 5(a) gives the varia-
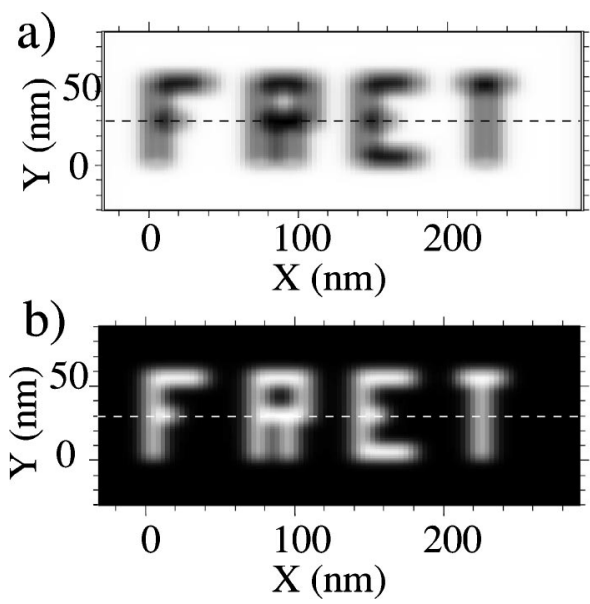

FIG. 5. Numerical simulation of fluorescence intensity maps for the donor (a) and the acceptor (b) molecules. The emission wavelengths are $\lambda_{0}=425 \mathrm{~nm}$ and $\lambda_{2}=500 \mathrm{~nm}$. The dipolar light source scans the sample in a parallel plane of $10 \mathrm{~nm}$ above the surface.
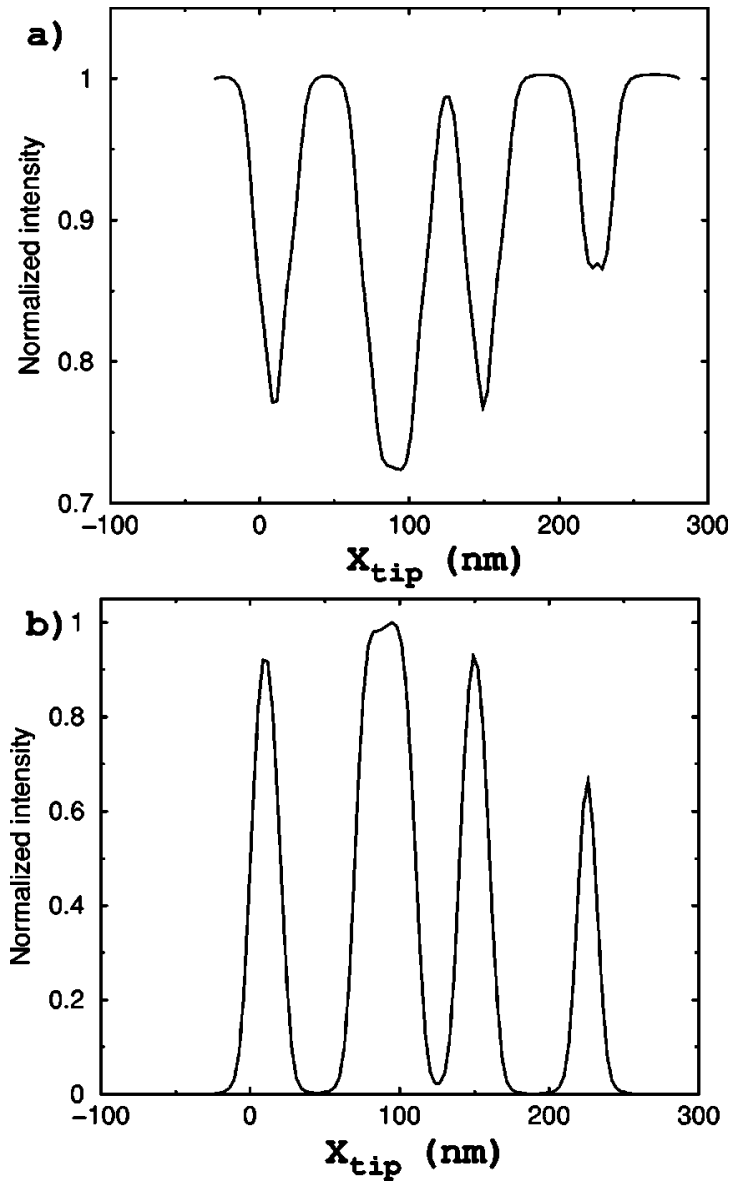

FIG. 6. Variation of the fluorescence signal intensity along the dashed line in Fig. 5. (a) Donor fluorescence and (b) acceptor fluorescence.

tion of the donor fluorescent signal when the probe-tip is raster scanned over the FRET pattern (wavelength $\lambda_{0}$ $=425 \mathrm{~nm}$ ). The dark region reveals the donor fluorescence decay that happens when the tip overhangs regions with much stronger concentration of acceptor molecules. We also note a simple image-object relation between this intensity map and the initial FRET pattern. This effect originates from the rapid spatial variation (in $R^{-3}$ ) of the coupling between chromophores pairs. The symmetrical fluorescence map is given in Fig. 5(b), where we show the acceptor fluorescent signal variation computed by scanning the same sample area (wavelength $\lambda_{2}=500 \mathrm{~nm}$ ). In this case, the FRET pattern occurs with a bright contrast that indicates the expected fluorescence increase generated by the acceptor molecules.

These simulations demonstrate the possibility to investigate FRET mechanisms at the nanometer scale. Moreover, in spite of several restrictive hypotheses, this model reproduces very well both the acceptor fluorescence and the donoracceptor energy transfer. Cross sections presented in Fig. 6 illustrate this energy transfer. These results are in good agreement with the experimental data obtained by Kirsch et al. (see, e.g., Fig. 1 in Ref. [10]).

\section{Beyond the pointlike probe approximation}

In the study of FRET using a SNOM, the proximity of the tip with the chromophores can significantly influence the re- 
sponse of the system. While the dipolar nature of the illumination field remains valid in that case, the coupling between the chromophores and the SNOM tip body can noticeably modify the decay rate of the different levels depicted in Fig. 1. As a matter of fact, the energy transfer is governed by a dipole-dipole coupling, which is strongly sensitive to its environment $[19,27,28]$. This coupling depends on the relative position between each chromophore and the tip, as well as the tip material and configuration. For example, in the experiment developed by Marti et al., a dielectric tip coated with a thin metal layer is used [9].

As previously mentioned, electrodynamics theory demonstrates that the coupling to the environment is fully included in the field-susceptibility tensor $\mathcal{S}\left(\mathbf{r}, \mathbf{r}^{\prime}, \omega\right)$ associated with the surrounding (the tip-surface junction in this case) [29]. The modified spontaneous emission rate for each chromophore in the complex surrounding can also be determined from this field susceptibility tensor [39,40]:

$$
\Gamma_{i}=\frac{2}{\hbar} \operatorname{Im}\left\{\boldsymbol{\mu}_{i} \cdot \mathcal{S}\left(\mathbf{r}_{m}, \mathbf{r}_{m}, \omega_{i}\right) \cdot \boldsymbol{\mu}_{i}\right\},
$$

where $\mathbf{r}_{m}$ denotes an arbitrary molecule location. In free space, this decay rate reduces to Eq. (43).

The field-susceptibility tensor can be computed by solving numerically the Dyson equation,

$$
\begin{aligned}
\mathcal{S}\left(\mathbf{r}_{i}, \mathbf{r}_{j}, \omega\right)= & \mathbf{T}\left(\mathbf{r}_{i}, \mathbf{r}_{j}\right)+\mathbf{S}_{s}\left(\mathbf{r}_{i}, \mathbf{r}_{j}, \omega\right)+\sum_{k=1}^{n} \chi_{k}\left(\mathbf{r}_{k}, \omega\right) \\
& \times\left[\mathbf{T}\left(\mathbf{r}_{i}, \mathbf{r}_{k}\right)+\mathbf{S}_{s}\left(\mathbf{r}_{i}, \mathbf{r}_{k}, \omega\right)\right] \cdot \mathcal{S}\left(\mathbf{r}_{k}, \mathbf{r}_{j}, \omega\right),
\end{aligned}
$$

associated with the discretized complex surrounding (the tipsurface junction in this case). In Eq. (45) the tip has been discretized with $n$ meshes centered at $\mathbf{r}_{k}, \mathbf{k}=1, \ldots, n$. The volume of each discretization cell is $V_{k}$, and the dielectric function $\epsilon\left(\mathbf{r}_{k}, \omega\right)$ of the tip enters in the definition of the susceptibility

$$
\chi_{k}\left(\mathbf{r}_{k}, \omega\right)=\left[\epsilon\left(\mathbf{r}_{k}, \omega\right)-\epsilon_{0}\right) V_{k} .
$$

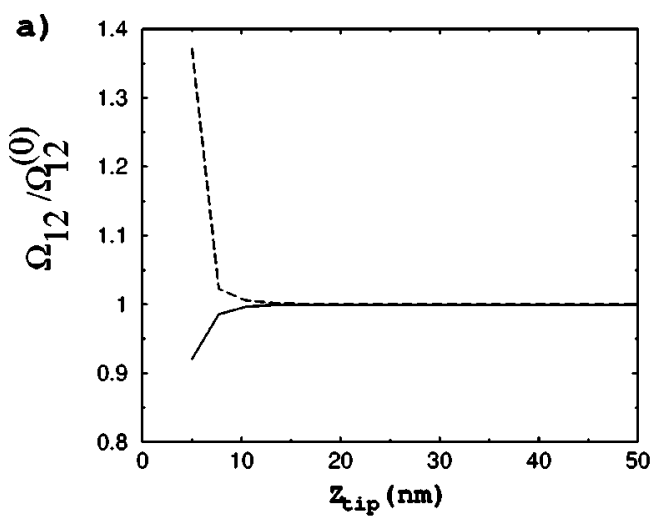

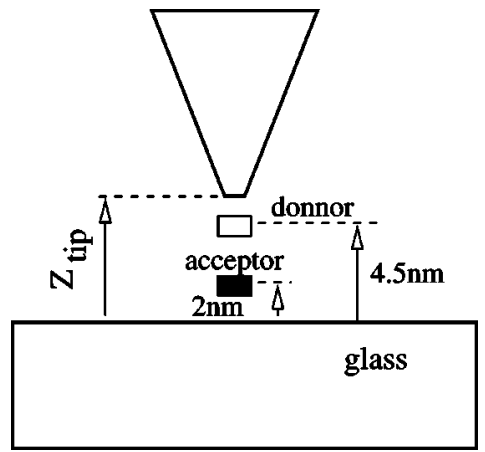

FIG. 7. Configuration under study, one single-donor dye is excited with an aluminum coated tip. FRET then occurs between this donor and a single acceptor molecule. The tip core is composed of a silica pyramid (100 nm height) with four sides coated with a layer of $10 \mathrm{~nm}$ thick aluminum.

The tip need not be homogeneous. For example, in the following simulations it is made of dielectric and metal. Note in Eq. (45) the tensor $\mathbf{S}_{\mathrm{s}}$, which accounts for the surface in the complex surrounding [see Eq. (42)].

To assess the influence of the complex surrounding formed by the the tip-surface junction on the Förster transfer, we consider the system depicted in Fig. 7. One single-donor dye is excited by the near-field of a pyramidal dielectric tip, with 10-nm aluminum coating. FRET can then occur between this single donor and a single acceptor placed at some distances. The fluorescence intensity is detected below the glass surface (Fig. 6, this configuration is similar to the experimental one studied in Ref. [9]).

Image calculations proceed therefore with the two following steps.

(i) Dyson equation (45) is solved for a given tip-sample configuration for both the selected wavelengths, and the fluorescence decay rates $\Gamma_{a_{1}}, \Gamma_{a_{2}}, \Gamma_{b_{1}}, \Gamma_{b_{2}}$ as well as the coupling parameters $\Omega_{12}$ and $\gamma_{12}$ are determined at the molecules (donor and acceptor) locations.

(ii) These parameters are then introduced in Eqs. (31) and (32) to obtain the fluorescence signals for the corresponding tip-sample configuration.

To simulate an approach curve or a complete image, the

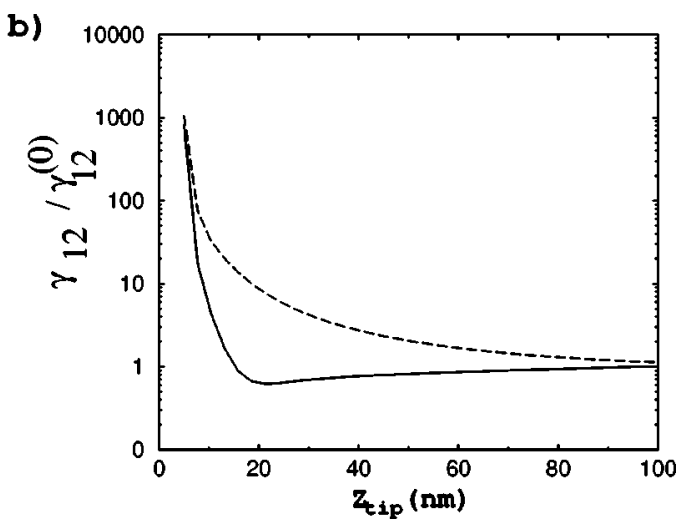

FIG. 8. Simulation of the variation of the dipole-dipole coupling coefficient as a function of the tip height above the surface. The dipole-dipole strength (a) and the cooperative decay (b) are represented for two dipole transition moments oriented along the $x$ axis (solid line) or along the $z$ axis (dotted line). The parameters have been normalized to their value calculated without the presence of the tip. 


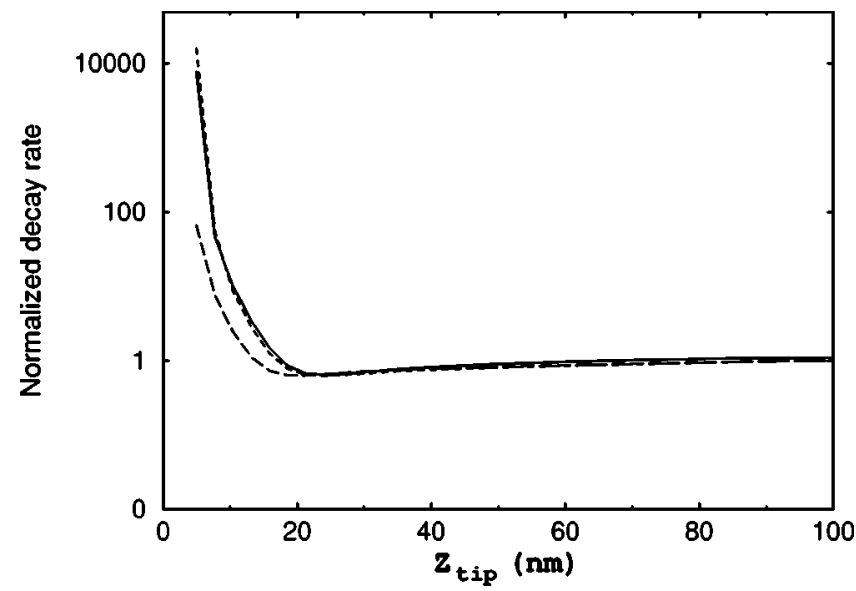

FIG. 9. Fluorescence decay rates $\Gamma_{a_{1}}$ (solid line), $\Gamma_{b_{1}}$ (dotted line), and $\Gamma_{b_{2}}$ (dashed line) as a function of the tip height above the surface. The dipole transition moments are oriented along the $x$ axis.

above procedure is repeated for the different tip-sample configurations.

The evolution of the molecular parameters as a function of the tip altitude $Z_{\text {tip }}$ above the surface (see Fig. 7) is shown in Figs. 8 and 9. Note the enhancement of both, levels decay rates $\Gamma_{a_{1}}, \Gamma_{b_{1}}, \Gamma_{b_{2}}$ and the cooperative dipole-dipole decay rate $\gamma_{12}$, when the tip is located at the immediate proximity of the molecules. On the other hand, the dipole-dipole strength $\Omega_{12}$ can either increase or decrease depending on mutual dipolar orientations. Consequently, when the tip scans over the surface at a short tip-sample distance $Z_{t i p}$, these different proximity effects can produce significant modifications of the FRET signal.

\section{CONCLUDING REMARKS}

The formalism presented in this paper allows the investigation at a molecular level of the fluorescence resonant energy transfer in complex geometries. This approach includes a nonperturbative quantum treatment of the active molecular levels. It can also handle the complex and rapidly varying field distributions associated with an intricate surrounding. The combination of these two features renders the method particularly powerful for the accurate analysis of FRET experiments performed with local probe techniques.

As an illustration of the method, experimental images recorded with near-field optical microscopy were accurately reproduced. Further, the different contrast mechanisms evidenced in the numerical simulations of FRET images indicate that an extremely high spatial resolution can be achieved with this technique.

Finally, calculations taking into account a complex surrounding including a large illumination probe tip reveal the magnitude of the perturbations imposed by this surrounding on the FRET rate. In some specific configurations, these perturbations can, however, be changed in a controlled manner, thereby generating new classes of experiments at the molecular level, where the Forster energy transfer rate can be modulated [20]. The formalism presented here could provide an efficient tool for optimizing such experimental configurations.
[1] T. Förster, Ann. Phys. (Leipzig) 2, 55 (1948).

[2] J. Barber, Rep. Prog. Phys. 41, 1157 (1978).

[3] V. Sundstrom and R. van Grondelle, J. Opt. Soc. Am. B 7, 1596 (1990).

[4] M. Berggren, A. Dodabalapur, R.E. Slusher, and Z. Bao, Synth. Met. 91, 65 (1997).

[5] P.R. Selvin, Nat. Struct. Biol. 7, 730 (2000).

[6] L. Matyus, J. Photochem. Photobiol., B 12, 323 (1992).

[7] N.P. Mahajan et al., Nat. Biotechnol. 16, 547 (1998).

[8] M.G. Xu et al., Scanning 23, 9 (2001).

[9] R. Brunner et al., Surf. Interface Anal. 25, 492 (1997).

[10] A.K. Kirsch, V. Subramaniam, A. Jenei, and T. Jovin, J. Microsc. 194, 448 (1999).

[11] G.T. Shubeita et al., Appl. Phys. Lett. 74, 3453 (1999).

[12] S.A. Vickery and R.C. Dunn, Biophys. J. 76, 1812 (1999).

[13] S.K. Sebatskii et al., J. Exp. Theor. Phys. 90, 769 (2000).

[14] S.K. Sebatskii, G.T. Shubeita, and G. Dietler, Opt. Commun. 188, 41 (2001).

[15] X.S. Xie and R.C. Dunn, Science (Washington, DC, U.S.) 265 , 361 (1994).

[16] W.L. Barnes, J. Mod. Opt. 45, 661 (1998).

[17] R. Chang, W. Fann, and S.H. Lin, Appl. Phys. Lett. 69, 2338 (1996).

[18] J. Azoulay, A. Débarre, A. Richard, and P. Tchénio, Europhys. Lett. 51, 374 (2000).
[19] G.S. Agarwal and S. Dutta Gupta, Phys. Rev. A 57, 667 (1998).

[20] P. Andrew and W.L. Barnes, Science (Washington, DC, U.S.) 290, 785 (2000).

[21] C.E. Finlayson, D.S. Ginger, and N.C. Greenham, Chem. Phys. Lett. 338, 83 (2001).

[22] C. Girard and A. Dereux, Rep. Prog. Phys. 59, 657 (1996).

[23] C. Cohen-Tannoudji, J. Dupont-Roc, and G. Grynberg, Processus d'interaction Entre Photons et Atomes (InterEditions, Paris, 1988).

[24] E. Paule and P. Reineker, J. Phys. Chem. B 105, 4293 (2001).

[25] P. Das and H. Metiu, J. Phys. Chem. 89, 4680 (1982).

[26] J. Courtois, J. Courty, and J.C. Mertz, Phys. Rev. A 53, 1862 (1996).

[27] C.R. Bennett, J.B. Kirk, and M. Babiker, Phys. Rev. A 63, 63812 (2001).

[28] R.L. Hartman and P.T. Leung, Phys. Rev. B 64, 193308 (2001).

[29] G.S. Agarwal, Phys. Rev. A 11, 230 (1975).

[30] G.S. Agarwal, Phys. Rev. A 12, 1475 (1975).

[31] J. Mahanty and B. W. Ninham, Dispersion Forces (Academic, London, 1976).

[32] O.J.F. Martin, C. Girard, and A. Dereux, J. Opt. Soc. Am. A 13, 1801 (1996).

[33] U.C. Fischer, J. Koglin, and H. Fuchs, J. Microsc. 176, 231 (1994). 
[34] G. Colas des Francs, C. Girard, and A. Dereux, J. Chem. Phys. 117, 4659 (2002).

[35] C. Chicanne et al., Phys. Rev. Lett. 88, 97402 (2002).

[36] P. Gay-Balmaz and O. Martin, Opt. Commun. 184, 37 (2000).

[37] F. H'Dhili, R. Bachelot, G. Lerondel, D. Barchiesi and P.
Royer, Appl. Phys. Lett. 79, 4021 (2001).

[38] Y. Chen and A. Pépin, Electrophoresis 22, 187 (2001).

[39] J.M. Wylie and J.E. Sipe, Phys. Rev. A 30, 1185 (1984).

[40] C. Girard, O.J.F. Martin, and A. Dereux, Phys. Rev. Lett. 75, 3098 (1995). 